\title{
Identification of key genes and crucial pathways for major depressive disorder using peripheral blood samples and chronic unpredictable mild stress rat models
}

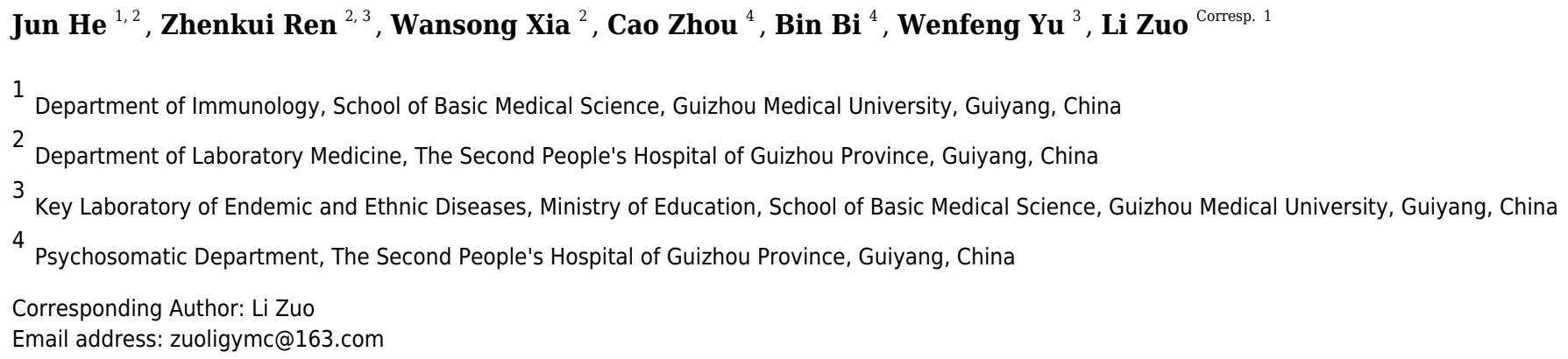

Background. Accurate diagnosis of major depressive disorder (MDD) remains difficult, and one of the key challenges in diagnosing MDD is the lack of reliable diagnostic biomarkers. The objective of this study was to explore gene networks and identify potential biomarkers for MDD. Methods. In the present study, we performed a comprehensive analysis of the mRNA expression profiles using blood samples of four patients with MDD and four controls by RNA sequencing. Differentially expressed genes (DEGs) were screened, and functional and pathway enrichment analyses were performed using the Database for Annotation, Visualization, and Integrated Discovery. All DEGs were inputted to the STRING database to build a PPI network, and the top 10 hub genes were screened using the cytoHubba plugin of the Cytoscape software. The relative expression of 10 key genes was identified by quantitative real-time polymerase chain reaction (qRT-PCR) of blood samples from 50 MDD patients and 50 controls. Plasma levels of SQSTM1 and TNF $\alpha$ were measured using an enzyme-linked immunosorbent assay in blood samples of 44 MDD patients and 44 controls. A sucrose preference test was used to evaluate depression-like behavior in chronic unpredictable mild stress (CUMS) model rats. Immunofluorescence assay and western blotting were performed to study the expression of proteins in the brain samples of CUMS model rats Results. We identified 247 DEGs that were closely associated with MDD. Gene ontology analyses suggested that the DEGs were mainly enriched in negative regulation of transcription by RNA polymerase II promoter, cytoplasm, and protein binding. Moreover, Kyoto Encyclopedia of Genes and Genomes pathway analysis suggested that the DEGs were significantly enriched in the MAPK signaling pathway. Ten hub genes were screened through the PPI network, and qRT-PCR assay revealed that one and six genes were downregulated and upregulated, respectively; 
however, SMARCA2, PPP3CB, and RAB5C were not detected. Pathway enrichment analysis for the 10 genes showed that the mTOR signaling pathway was also enriched. A strong positive correlation was observed between SQSTM1 and TNF $\alpha$ protein levels in patients with MDD. LC3 II and SQSTM1 protein levels were increased in the CUMS rat model; however, p-mTOR protein levels were decreased. The sucrose preference values decreased in the CUMS rat model. Conclusions. We identified 247 DEGs and constructed an MDD-specific network; thereafter, 10 hub genes were selected for further analysis. Our results provide novel insights into the pathogenesis of MDD. Moreover, SQSTM1, which is related to autophagy and inflammatory reactions, may play a key role in MDD. SQSTM1 may be used as a promising therapeutic target in MDD; additionally, more molecular mechanisms have been suggested that should be focused on in future in vivo and in vitro studies. 
1 Identification of key genes and crucial pathways for major depressive disorder using peripheral blood samples and chronic unpredictable mild stress rat models

Jun $\mathrm{He}^{1,2}$, Zhenkui Ren ${ }^{2,3}$, Wansong Xia ${ }^{2}$, Cao Zhou ${ }^{4}$, Bin $\mathrm{Bi}^{4}$, Wenfeng $\mathrm{Yu}^{3}$, Li Zuo ${ }^{1 *}$

${ }^{1}$ Department of Immunology, School of Basic Medical Science, Guizhou Medical University, Guiyang, 550004, China;

${ }^{2}$ Department of Laboratory Medicine, The Second People's Hospital of Guizhou Province, Guiyang, 550002, China;

${ }^{3}$ Key Laboratory of Endemic and Ethnic Diseases, Ministry of Education, School of Basic Medical Science, Guizhou Medical University, Guiyang, 550004, China;

${ }^{4}$ Psychosomatic Department, The Second People's Hospital of Guizhou Province, Guiyang, 550002, China

* Correspondence Author: Li Zuo, zuoligymc@163.com

Keywords: SQSTM1, Major depressive disorder, Inflammation, PPI network analysis, CUMS, Autophagy

\section{ABSTRACT}

Background. Accurate diagnosis of major depressive disorder (MDD) remains difficult, and one of the key challenges in diagnosing MDD is the lack of reliable diagnostic biomarkers. The objective of this study was to explore gene networks and identify potential biomarkers for MDD.

Methods. In the present study, we performed a comprehensive analysis of the mRNA expression profiles using blood samples of four patients with MDD and four controls by RNA sequencing. Differentially expressed genes (DEGs) were screened, and functional and pathway enrichment analyses were performed using the Database for Annotation, Visualization, and Integrated Discovery. All DEGs were inputted to the STRING database to build a PPI network, and the top 10 hub genes were screened using the cytoHubba plugin of the Cytoscape software. The relative expression of 10 key genes was identified by quantitative real-time polymerase chain reaction (qRT-PCR) of blood samples from 50 MDD patients and 50 controls. Plasma levels of SQSTM1 and TNF $\alpha$ were measured using an enzyme-linked immunosorbent assay in blood samples of 44 MDD patients and 44 controls. A sucrose preference test was used to evaluate depression-like behavior in chronic unpredictable mild stress (CUMS) model rats. Immunofluorescence assay and western blotting were performed to study the expression of proteins in the brain samples of CUMS 
model rats.

Results. We identified 247 DEGs that were closely associated with MDD. Gene ontology analyses suggested that the DEGs were mainly enriched in negative regulation of transcription by RNA polymerase II promoter, cytoplasm, and protein binding. Moreover, Kyoto Encyclopedia of Genes and Genomes pathway analysis suggested that the DEGs were significantly enriched in the MAPK signaling pathway. Ten hub genes were screened through the PPI network, and qRT-PCR assay revealed that one and six genes were downregulated and upregulated, respectively; however, SMARCA2, PPP3CB, and RAB5C were not detected. Pathway enrichment analysis for the 10 genes showed that the mTOR signaling pathway was also enriched. A strong positive correlation was observed between SQSTM1 and TNFa protein levels in patients with MDD. LC3 II and SQSTM1 protein levels were increased in the CUMS rat model; however, p-mTOR protein levels were decreased. The sucrose preference values decreased in the CUMS rat model.

Conclusions. We identified 247 DEGs and constructed an MDD-specific network; thereafter, 10 hub genes were selected for further analysis. Our results provide novel insights into the pathogenesis of MDD. Moreover, SQSTM1, which is related to autophagy and inflammatory reactions, may play a key role in MDD. SQSTM1 may be used as a promising therapeutic target in MDD; additionally, more molecular mechanisms have been suggested that should be focused on in future in vivo and in vitro studies.

\section{INTRODUCTION}

Major depressive disorder (MDD) is a highly prevalent mental disorder that causes suicidal death and is a significant healthcare burden worldwide (Park \& Jung 2019). However, there are no effective methods for the treatment and prevention of MDD. At present, diagnosis of MDD depends on the patient's self-reported symptoms and a clinician's evaluation (Jain et al. 2016). For this reason, differential diagnosis is difficult and often results in misdiagnosis and missed diagnosis. Therefore, it is beneficial to improve diagnosis and treatment by exploring the underlying molecular mechanisms and identifying novel biomarkers for this disorder (Cui et al. 
2016).

Currently, the pathogenesis of MDD is still unclear, which is generally thought to be associated with complex factors, including between social pressure (Juan et al. 2014), genetic, epigenetic factors, and their interaction (Kathleen et al. 2016; Liou et al. 2013). One of important roles in the pathogenesis is genetic factors (Barbu et al. 2020). For example, 5-hydroxytryptamine (5-HT) is a very crucial monoamine neurotransmitter that regulates a variety of functions, such as locomotor activity, cognition, emotion, food intake (T. et al. 1976). 5-HT was focused upon as a key molecular in the pathogenesis of MDD (Sa et al. 2012). However, MDD is a common mental disorder, which is regulated by a complex gene network (Feng et al. 2020). Hence, screening specific gene expression profiles are promising for the use of the diagnosis and treatment of MDD.

Gene expression profiling analysis is widely used to investigate the correlations between the occurrence of diseases and differential gene expression(Cooper-Knock et al. 2012). Blood mRNA profiles are reportedly significantly altered and are associated with disease outcomes in diseases of the nervous system (Laing et al. 2019); therefore, gene expression profiling analysis is a promising method for screening disease biomarkers. Substantial efforts have been undertaken to report the abnormal expression of some genes and pathways associated with MDD. However, most of these results have only identified differentially expressed genes (Yi et al. 2012), and there has been an absence of further exploration of the underlying biological molecular mechanisms; this limited approach may have inhibited the development of effective biomarkers. Thus, investigation of the molecular mechanisms of differentially expressed genes, which may allow definitive MDD diagnosis, is urgently required.

Hence, the present study evaluated potential diagnostic biomarkers and molecular mechanisms associated with MDD. Further, differential expression genes were screened between MDD patients and healthy controls. Moreover, the molecular functions of 147 differential expression genes were analyzed using gene ontology (GO) enrichment and KEGG enrichment analysis. In addition, the top 10 hub genes screened via protein-protein interaction (PPI) network were identified through 50 MDD patients and 50 healthy controls. Especially, one of the key genes was SQSTM1, which 
86

was associated with autophagy and was upregulated in the MDD patient's plasma. This finding was further confirmed in CUMS rat model. Consequently, the results of the present study indicated that SQSTM1 may play a crucial role through involved in the regulation of autophagy.

\section{MATERIALS AND METHODS}

\section{mRNA isolation and RNA sequencing (RNA-Seq)}

Total RNA was extracted using TRIzol ${ }^{\circledR}$ reagent (Invitrogen; Thermo Fisher Scientific, Inc.) from the blood samples of four MDD patients and four controls. We received WRITTEN informed consent from participants of our study. mRNA profiles were obtained using high-throughput nextgeneration sequencing, which was performed using an Illumina HiSeq 4000 platform, Genes with $p<0.001$ were regarded as differentially expressed mRNAs (DEGs), and the gene expression was showed as the raw counts (Novogene Bioinformatics Institute, Beijing, China).

\section{Functional and pathway enrichment analysis}

The Database for Annotation, Visualization, and Integrated Discovery (DAVID) was used to enrich the differentially expressed mRNAs (DEGs) GO and perform Kyoto Encyclopedia of Genes and Genomes (KEGG) pathway analysis. DAVID is a web tool that allows users to integrate and analyze biological data, including biological process (BP), cellular component (CC) and molecular function (MF) terms (Da et al. 2007). $P<0.05$ was considered statistically significant.

\section{PPI network construction and module analysis}

The PPI network was built by STRING, which is a useful online database for constructing a protein-protein interaction network (Franceschini et al. 2013). In order to evaluate their interactive associations, all DEGs were inputted to the STRING database, and chose a larger score subnetwork to further analysis through the Cytoscape software (a visual analytics platform for PPI networks). The top 10 hub genes were screened using cytoHubba plugin. The algorithms applied to identify hub genes were the maximal clique centrality (MCC) and Degree. 


\section{Validation analysis of hub genes}

115 The key genes were identified from the PPI network by cytoHubba plugin. Details of these key 116 genes, such as names, abbreviations and functions were obtained by searching NCBI.

117 Subsequently, the relative expression of 10 key genes was identified using quantitative real-time 118 PCR (qRT-PCR) of blood samples from 50 MDD patients and 50 controls. In brief, total RNA was 119 extracted from the blood samples using TRIzol reagent (Invitrogen) following the manufacturer's 120 instructions. StarScript II First-strand cDNA Synthesis Mix with gDNA Remover kit, which was

121

122

123

124

125

126

\section{7}

128

129

130

131

132

133

134

135

136

137

138

139

obtained from GenStar, was used to synthesize cDNA $\left(45^{\circ} \mathrm{C}\right.$ for $30 \mathrm{~min}$ and $85{ }^{\circ} \mathrm{C}$ for $5 \mathrm{~min}$ ). SYBR-Green SuperMix (Vazyme Biotech Co., Ltd.) was used to amplify the cDNA as follows: $94{ }^{\circ} \mathrm{C}$ for $3 \mathrm{~min}, 40$ cycles of $94{ }^{\circ} \mathrm{C}$ for $7 \mathrm{~s}$, and $60{ }^{\circ} \mathrm{C}$ for $30 \mathrm{~s}$. The relative expression of $10 \mathrm{key}$ genes was calculated by the $2-\Delta \Delta \mathrm{Ct}$ method. $H P R T$ was used as an internal control. Table 1 . Primer sequences.

\section{Plasma SQSTM1 and TNFa}

SQSTM1 is involved in the regulation of autophagy and inflammation (Chu et al. 2020; Yao et al. 2020). The serum level of TNF $\alpha$ was increased in MDD patients, which was associated with depressive symptoms (Alvarez-Mon et al. 2021). We thus examined the expression of these two proteins in the plasma. Blood samples were collected from the 44 patients with MDD and 44 healthy controls. Plasma was separated from whole blood and stored at $-80^{\circ} \mathrm{C}$ before use. Plasma levels of total SQSTM1 and TNF $\alpha$ were measured using an enzyme linked immunosorbent assay (ELISA) according to the manufacturer's instructions; these two ELISA kits were purchased from Fine Test (Wuhan Fine Biotech Co., Ltd) and Elabscience (Elabscience Biotechnology Co., Ltd). Correlation analysis between SQSTM1 and TNF $\alpha$ was performed using the R software, version 3.5.3.

\section{Chronic unpredictable mild stress (CUMS) model was established}


140 The present study was approved by the Animal Care and Welfare Committee and the Ethics

141 Committee of Guizhou 2nd Provincial People's Hospital (permit no. 2020[06]). Twenty four

142 female Sprague-Dawley (SD) rats weighing 180-220g, obtained from the Experimental Animals

143 Center at Guizhou Medical University. Rats were bred under standard conditions (12:12-h

144 light/dark cycle, humidity $55-60 \%, 20-25^{\circ} \mathrm{C}$ ) for adaption. Twelve rats were housed in each group

145 (six rats were used to Immunofluorescence assay, six rats were used to western blotting).

146 According to previous studies (Yanxia et al. 2018a; Yanxia et al. 2018b), CUMS depression-like

147 behavior model was established in SD rats, twelve rats underwent 21 days of CUMS procedure-

148 induced as previously described (Zhang et al. 2019), with the control group being without any

149 treatment. During a 21 days period, the CUMS group were exposed to different stimuli (food and

150 water deprivation for $24 \mathrm{~h}$, immobilization $2 \mathrm{~h}$, level shaking $5 \mathrm{~min}$ and tail clamping). To ensure

151 the unpredictability of the occurrence of stimulation, these stressors were changed randomly as

152 one stressor per day in rats. Model rats were evaluated by two trained experimenters by a double

153 blind way.

154

155 Sucrose Preference Test (SPT)

156 A sucrose Preference Test (SPT) was used to evaluate the depression-like behavior of model rats, 157 the procedure of SPT was performed as described previous method (Chen et al. 2019; Meng-Ying et al. 2018). First, rats in each group were trained to adapt to $1 \%$ sucrose solution (weight in volume [w/v]) with 2 bottles of $1 \%$ sucrose solution for the first day. After $24 \mathrm{~h}$ later, one bottle of $1 \%$ sucrose was replaced with tap water and continued adapting for $24 \mathrm{~h}$. Before each test, rats were deprived of food and water for $24 \mathrm{~h}$. During the $1 \mathrm{~h} \mathrm{SPT}$, rats were caged alone and, had a chance to access one bottle containing $200 \mathrm{~mL}$ of sucrose solution $(1 \% \mathrm{w} / \mathrm{v})$ and one bottle containing $200 \mathrm{~mL}$ of water, respectively. The positions of two bottles were randomly assigned to prevent place preference, each bottle was weighed before and after the test. According to the formula: sucrose preference $=1 \%$ sucrose solution consumption/total amount of liquid intake $\times 100 \%$, the sucrose preference was calculated as the percentage. Next, the rats were returned to their cages 
and allowed free access to food and water.

\section{Immunofluorescence assay}

After the CUMS rat model was successfully established, the rats were euthanized and perfused transcardially with 4\% paraformaldehyde. $\mathrm{CO} 2$ inhalation was used to conduct rat euthanasia; rats were placed into enclosed cages for $5 \mathrm{~min}, \mathrm{CO} 2(100 \%)$ was then added to the cages at a displacement rate of $30 \%$ volume/min. Death was confirmed via decapitation after euthanasia after euthanasia. The rat brains were mounted on a freezing $\left(-80^{\circ} \mathrm{C}\right)$ microtome and cut into $30 \mu \mathrm{m}$ sections. Frozen sections were blocked with 5\% normal goat serum (Sigma-Aldrich; Merck $\mathrm{KGaA}$ ) and permeabilized with $0.3 \%$ Triton $\mathrm{X}-100$ for $1 \mathrm{~h}$ at room temperature. Next, the slices were incubated with primary antibodies against LC3 (1:200; cat. no. 4108S; Cell Signaling Technology, Inc.) and SQSTM1 (1:200; cat. no. 23214; Cell Signaling Technology, Inc.) at $4^{\circ} \mathrm{C}$ overnight. Alexa Fluor ${ }^{\circledR}$ 568-conjugated goat anti-rabbit IgG $(\mathrm{H}+\mathrm{L})$ cross-adsorbed secondary antibody (1:100; cat. no. A-11011) was purchased from Thermo Fisher Scientific and was used to bind the primary antibody for $1 \mathrm{~h}$ at room temperature. The slices were washed three times with PBS, before the nuclei were counterstained with DAPI (Beyotime Institute of Biotechnology) for 5 min at room temperature. Using a confocal laser scanning microscope (Olympus FV 1000; Olympus Corporation) to acquire image. Twenty randomly selected fields/sections were analyzed

\section{Western blotting}

The brain hippocampal tissues of SD rats were collected and homogenized in RIPA buffer $(1 \mathrm{mg} / 10 \mu \mathrm{L}$; Thermo Fisher Scientific, Inc.) containing a protease inhibitor cocktail (Sigma-Aldrich; Merck KGaA) according to a previously described method (Wang et al. 2020). After the lysis buffer was kept for $2 \mathrm{~h}$ on ice, being centrifuged at $12000 \mathrm{rpm}$ for $20 \mathrm{~min}$. The protein contents were determined by a bicinchoninic acid assay kit (cat. no. P0009; Beyotime Institute of Biotechnology), next, $25 \mu \mathrm{g}$ proteins were separated by 12\% SDS-PAGE and transferred onto nitrocellulose membranes. $5 \%$ nonfat milk was used to block the membrane at room temperature 
194

195

196

197

198

199

200

201

202

203

204

205

206

207

208

209

210

211

212

213

214

215

216

217

218

219

220 2

for $2 \mathrm{~h}$, before incubated with primary antibodies against the autophagy-related proteins from Cell Signaling Technology company: phosphorylated (p)-mTOR (1:1,000; cat. no. 5536S), mTOR (1:1,000; cat. no. 2972S), SQSTM1 (1:1,000; cat. no. 23214), light chain (LC)3II/I (1:1,000; cat. no. $4108 \mathrm{~S}), \beta$-actin $(1: 1,000$; cat. no. $3700 \mathrm{~S})$, and TNFa $(1: 1,000$; cat. no. ab205587) from Abcam overnight at $4^{\circ} \mathrm{C}$. Subsequently, a horseradish peroxidase-conjugated secondary antibody $(1: 1,000$; cat. no. 7074S; Cell Signaling Technology, Inc.) was used to incubate membranes at room temperature for $2 \mathrm{~h}$, and the membranes were visualized by an enhanced chemiluminescence reagent kit (Amersham; Cytiva). Gray values of proteins were measured using ImageJ software (version 1.42q, National Institutes of Health) and normalized to that of $\beta$-actin, which was the internal control.

\section{Statistical analysis}

All statistical analyses were conducted using GraphPad Prism software 7.0 (GraphPad Software, Inc.), and data are shown as mean \pm standard deviation. Multiple groups comparison analysis was performed using one-way analysis of variance followed by Tukey's post hoc test; comparisons were made between the two groups through unpaired t-tests. $P<0.05$ was considered a statistically significant difference.

\section{Results}

\section{Differentially expressed genes identification}

RNA-seq analysis between the MDD and control blood samples revealed the DEGs (16272 genes Genes detected in this transcriptome study). We drew a heat map using four biological replicates for each group (Fig. 1A). The volcano plot showed that 180 mRNAs were significantly upregulated and 67 mRNAs were significantly downregulated according to $P$-value $<0.001$ (Fig. 1B).

\section{Functional and pathway enrichment analysis of DEGs}


221 To explore biological relevance of the 247 DEGs by DAVID database. GO analysis included BP, $222 \mathrm{MF}$, and CC. Our results suggested that the DEGs were mainly enriched in negative regulation of 223 transcription from RNA polymerase II promoter, in the cytoplasm, and in protein binding (Fig. 224 2A). Moreover, KEGG pathway analysis indicated that the DEGs were significantly enriched in 225 the MAPK signaling pathway (Fig. 2B).

226

227

\section{PPI network analysis of DEGs}

The interactions of 247 DEGs were explored using the STRING online database to investigate the PPI network likely associated with MDD. The obtained PPI network were visualized by the Cytoscape software (Fig. 3A). The top 10 hub genes were screened using the cytoHubba plugin (Fig. 3B). To better understand the functions of 10 hub genes, there is a need to perform a more systematic analysis through pathway enrichment in DAVID database (Fig. 3C). The following top 10 hub genes were identified from the PPI network using the cytoHubba plugin: $Y W H A Z$, SQSTM1, ITGB2, RAB1A, MYSM1, SMARCA2, PPP3CB, AKT2, TSC2, and RAB5C. Details of these key genes, such as names, abbreviations, and functions, are provided in Table 2

Validation analysis of the 10 hub genes

The mRNA expression of 10 hub genes were identified between the MDD and control blood samples by q-PCR. A total of 1 decreased gene and 6 increased genes were detected, however, SMARCA2, PPP3CB and RAB5C were not detected (Fig. 4).

\section{Positive correlation between the levels of SQSTM1 and TNFa}

Our results showed that the protein levels of SQSTM1 and TNF $\alpha$ were significantly increased in patients with MDD (Fig. 5A, B). Further analysis revealed a strong positive correlation was seen between SQSTM1 and TNFa protein levels (Fig. 5C). 
249

250

251

252

253

254

255

256

257

258

259

260

261

262

263

264

265

266

267

268

269

270

271

272

273

274

275

\section{Autophagy related proteins were detected in CUMS rat models}

SQSTM1 mRNA level is higher in MDD blood samples than control blood samples. To further explore SQSTM1 protein level changes in the CUMS rat models, it is important to identify the specific function of SQSTM1. The in vivo results from the present study demonstrated that SQSTM1 protein levels were increased in the CUMS rat models (Fig. 6A, D). Meanwhile, LC3, which is an autophagy activated marker, was upregulated in the CUMS rat model (Fig. 6B, C). mTOR a key signaling pathway associated with negatively regulating autophagy, was inhibited (Fig. 6E). SPT was used to evaluate depression-like behavior in the model rats (Fig. 6F).

\section{DISSCUSSION}

In the first part of our study, we screened 247 DEGs between the blood samples of four MDD patients and four controls. Based on the BP annotations, negative regulation of transcription by RNA polymerase II promoter, positive regulation of GTPase activity, intracellular signal transduction and cell-cell adhesion were suggested to be significantly involved in the pathologic processes of MDD. Of the CC annotations, the cytoplasm, cytosol, nucleoplasm and membrane were found to play important roles in MDD. According to the MF annotations, protein binding, cadherin binding involved in cell-cell adhesion, protein serine/threonine kinase activity and protein kinase activity were predicted to be significantly correlated with the occurrence and development ofMDD. Moreover, KEGG pathway analysis indicated that the DEGs were significantly enriched in the MAPK signaling pathway. MAPK pathways are involved in the induction of inflammatory factors(Inamdar et al. 2014). Further research found that TNF $\alpha$ is also upregulated in plasma of MDD, which is in agreement with a previous study (Li et al. 2013). Taken together, these findings shed light on the inflammatory factors that may be major contributors to MDD.

In the second part of our study, in order to evaluate their interactive associations, all DEGs were inputted to the STRING database to build the PPI network, and a subnetwork with a large score was chosen for further analysis using Cytoscape software. The top 10 hub genes were screened using the cytoHubba plugin. Further investigation of these key genes may reveal novel molecular 
276

277

278

279

280

281

282

283

284

285

286

287

288

289

290

291

292

293

294

295

296

297

mechanisms underlying MDD. Next, the mRNA expression of 10 hub genes was assessed in the blood samples of 50 MDD patients and 50 controls by qRT-PCR. One downregulated gene and six upregulated genes were detected; however, SMARCA2, PPP3CB and RAB5C were not detected. Uncovering the molecular functions of SQSTM1 deserved more attention in our study. SQSTM1 is a biomarker of autophagy, and is predicted to be associated with inflammatory factors(Lee et al. 2011). Moreover, the levels of SQSTM1 and TNF $\alpha$ were positively correlated based on the ELISA. Therefore, we speculated that SQSTM1 may be associated with the regulation of inflammatory reactions. Inflammation is supposed to play an important role in the pathophysiology of MDD(Marazziti et al. 2020).

To expore further details of the 10 hub genes, their functions were searched using NCBI. Our results showed that YWHAZ regulates spine maturation through the modulation of ARHGEF7 activity, and could be associated with depression in patients with Alzheimer's Disease (AD) (Yang et al. 2020). SQSTM1 might regulate the activation of NFKB1 by TNF-alpha, and the overexpression of SQSTM1 could ameliorate spatial learning and long-term memory, indicating that SQSTM1 is a potential diagnostic marker and therapeutic target. RAB1A is involved in the inflammatory signaling pathway(Song et al. 2020). Akt2 knockout led to more significantly anxiety and depressive behaviors than corresponding wild type mice; therefore, this might be a crucial factor in the pathophysiology of depression and anxiety(Leibrock et al. 2013). TSC2 mutations are related to the level of long-term depression via regulation of hippocampal synapse function(von der Brelie et al. 2006). The molecular mechanism of above five hub genes involved in MDD deserves further investigation. However, the relationship between the remaining five hub genes (MYSM1, ITGB2, SMARCA2, RAB5C, PPP3CB) and MDD have not been reported, it could be that these five hub genes could have potential function in MDD.

For a better understand the roles of 10 hub genes, there is a need to perform a more systematic analysis through pathway enrichment in DAVID database. In particular, we found that the mTOR, which is involved in the regulation of autophagy and inflammation signaling pathway (Chu et al. 2020; Yao et al. 2020) was enriched. Further study found that mTOR signaling pathway is 
303

304

305

306

307

308

309

310

311

312

313

314

315

316

317

318

319

320

321

322

323

324

325

326

327

328

329

inhibited in CUMS rat model, which is in agreement with the results reported previously (Zhang et al. 2020; Zhuo et al. 2020). SQSTM1, which was also upregulated in CUMS rat model, is associated with autophagy. Moreover, the expression of LC3II was increased in the CUMS rat model, which is consistent with the results reported previously (Zhang et al. 2020). Therefore, it is reasonable to speculate that SQSTM1 could play a crucial role through regulated autophagy in MDD.

\section{CONCLUSIONS}

In conclusion, this study was to explore the molecular function of DEGs via a comprehensive bioinformatics analysis, and to identify hub genes were involved in the development of MDD. Especially, the expression of 10 hub genes was confirmed between 50 MDD patients and 50 healthy controls, however, SMARCA2, PPP3CB and RAB5C were not detected. SQSTM1 was significantly focused on in the present study, which was associated with autophagy and inflammatory reactions. Further analysis revealed a strong positive correlation was seen between SQSTM1 and TNF $\alpha$ protein levels in the plasma of MDD. SQSTM1 may be used as a promising therapeutic target in MDD; additionally, several other molecular mechanisms have been suggested, which should be focused on in future in vivo and in vitro studies.

\section{ACKNOWLEDGEMENTS}

Not applicable.

\section{Funding}

This study was supported by grants from the National Natural Science Foundation of China (grant no. 81360199), Guizhou Provincial Key Discipline Construction Project (psychosomatic medicine), Special Grant for Central Government Supporting Local Science and Technology Development, Science and Technology Department of Guizhou Province [grant no. (2019) 4008], Science and Technology Plan Project of Guizhou Province (Basic Science and Technology Cooperation) [grant no. (2020)1Z060], the Science and Technology Fund Project of Guizhou Health and Health Commission (grant no. gzwjkj2019-1-039), and the Science and Technology Fund Project of Southwest Guizhou Autonomous Prefecture (grant no. 2019-1-10), Basic Science 
330 Technology Project of Guizhou Province [grant no. ZK [2021] 412]

331 Competing Interests

332 The authors declare that there are no conflicts of interest.

333 Authors' contributions

334 Jun He drafted the manuscript.

335 Li Zuo designed the study.

336 Zhenkui Ren and Wansong Xia performed the experiments.

337 Cao Zhou and Bin Bi contributed to the analysis of data

338 Wenfeng Yu contributed reagents/materials/analysis tools

339 Data Availability

340 The datasets used and/or analyzed during the current study are available from the Supplemental

341 File (the raw data of datasets)

342 REFERENCES

343 Alvarez-Mon MA, Gómez-Lahoz AM, Orozco A, Lahera G, Diaz D, Ortega MA, Albillos A, Quintero J, Aubá E, 344 Monserrat J, Alvarez-Mon M. 2021. Expansion of CD4 T Lymphocytes Expressing Interleukin 17 and Tumor Necrosis Factor in Patients with Major Depressive Disorder. J Pers Med. 11. 10.3390/jpm11030220.

Barbu MC, Shen X, Walker RM, Howard DM, Evans KL, Whalley HC, Porteous DJ, Morris SW, Deary IJ, Zeng Y, Marioni RE, Clarke TK, McIntosh AM. 2020.Epigenetic prediction of major depressive disorder. Mol Psychiatry 10. 10.1038/s41380-020-0808-3.

Chen B, Li J, Xie Y, Ming X, Li G, Wang J, Li M, Li X, Xiong L. 2019. Cang-ai volatile oil improves depressive-like behaviors and regulates DA and 5-HT metabolism in the brains of CUMS-induced rats. $J$ Ethnopharmacol 15. 10.1016/j.jep.2019.112088.

Chu Y, Chen W, Peng W, Liu Y, Xu L, Zuo J, Zhou J, Zhang Y, Zhang N, Li J, Liu L, Yao K, Gao G, Wang X, Han R, Liu C, Li Y, Zhou H, Huang Y, Ye Y. 2020. Amnion-Derived Mesenchymal Stem Cell ExosomesMediated Autophagy Promotes the Survival of Trophoblasts Under Hypoxia Through mTOR Pathway by the Downregulation of EZH2. Front Cell Dev Biol 11. 10.3389/fcell.2020.545852. 
neurodegenerative disease. Nat Rev Neurol 8. 10.1038/nrneurol.2012.156.

358

359

360

361

362

363

364

365

366

367

368

369

370

371

372

373

374

375

376

377

378

379

380

381

382

383

Cui X, Niu W, Kong L, He M, Jiang K, Chen S, Zhong A, Li W, Lu J, Zhang L. 2016. hsa_circRNA_103636: potential novel diagnostic and therapeutic biomarker in Major depressive disorder. Biomark Med 10. 10.2217/bmm2016-0130.

Huang DW, Sherman BT, Tan Q, Collins JR, Alvord WG, Roayaei J, Stephens R, Baseler MW, Lane HC, 2007. Lempicki RA. The DAVID Gene Functional Classification Tool: a novel biological module-centric algorithm to functionally analyze large gene lists. Genome Biol 8. 10.1186/gb-2007-8-9-r183.

Feng J, Zhou Q, Gao W, Wu Y, Mu R. 2020. Seeking for potential pathogenic genes of major depressive disorder in the Gene Expression Omnibus database. Asia Pac Psychiatry 12. 10.1111/appy.12379.

Franceschini A, Szklarczyk D, Frankild S, Kuhn M, Simonovic M, Roth A, Lin J, Minguez P, Bork P, von Mering C, Jensen LJ. 2013. STRING v9.1: protein-protein interaction networks, with increased coverage and integration. Nucleic Acids Res 41. 10.1093/nar/gks1094.

Inamdar A, Merlo-Pich E, Gee M, Makumi C, Mistry P, Robertson J, Steinberg E, Zamuner S, Learned S, Alexander R, Ratti E. 2014. Evaluation of antidepressant properties of the p38 MAP kinase inhibitor losmapimod (GW856553) in Major Depressive Disorder: Results from two randomised, placebo-controlled, double-blind, multicentre studies using a Bayesian approach. J Psychopharmacol 28. 10.1177/0269881114529377.

Jain N, Carmody T, Minhajuddin AT, Toups M, Trivedi MH, Rush AJ, Hedayati SS. 2016. Prognostic Utility of a Self-Reported Depression Questionnaire versus Clinician-Based Assessment on Renal Outcomes. Am J Nephrol. $10.1159 / 000448598$.

Li J, Tang G, Cheng K, Yang D, Chen G, Liu Z, Zhang R, Zhou J, Fang L, Fang Z, Du X, Xie P. 2014. Peripheral blood mononuclear cell-based metabolomic profiling of a chronic unpredictable mild stress rat model of depression. Mol Biosyst 10. 10.1039/c4mb00388h.

Saavedra K, Molina-Márquez AM, Saavedra N, Zambrano T, Salazar LA. 2016. Epigenetic Modifications of Major Depressive Disorder. Int J Mol Sci 5. 10.3390/ijms17081279.

Laing EE, Möller-Levet CS, Dijk DJ, Archer SN. 2019. Identifying and validating blood mRNA biomarkers for acute and chronic insufficient sleep in humans: a machine learning approach. Sleep 42. 10.1093/sleep/zsy186.

Lee HM, Shin DM, Yuk JM, Shi G, Choi DK, Lee SH, Huang SM, Kim JM, Kim CD, Lee JH, Jo EK. 2011. Autophagy

Peer) reviewing PDF | (2020:12:56643:2:2:NEW 2 Jun 2021) 
negatively regulates keratinocyte inflammatory responses via scaffolding protein p62/SQSTM1. J Immunol 15. 10.4049/jimmunol.1001954.

Leibrock C, Ackermann TF, Hierlmeier M, Lang F, Borgwardt S, Lang UE. 2013. Akt2 deficiency is associated with anxiety and depressive behavior in mice. Cell Physiol Biochem 32. 10.1159/000354478.

Li Z, Qi D, Chen J, Zhang C, Yi Z, Yuan C, Wang Z, Hong W, Yu S, Cui D, Fang Y. 2012. Venlafaxine inhibits the upregulation of plasma tumor necrosis factor-alpha (TNF- $\alpha$ ) in the Chinese patients with major depressive disorder: a prospective longitudinal study. Psychoneuroendocrinology 38. 10.1016/j.psyneuen..05.005.

Liou YJ, Chen TJ, Cheng CY, Younger W, Yu Y, Hong CJ, Tsai SJ. 2013. Brain-derived Neurotrophic Factor Genetic Variants are Associated with Major Depression Susceptibility and Serotonin Reuptake Inhibitor Antidepressant Treatment Response in Taiwanese. Current Pharmacogenomics and Personalized Medicine 11. $10.2174 / 1875692111666131211235804$.

Marazziti D, Parra E, Palermo S, Barberi FM, Buccianelli B, Ricciardulli S, Cappelli A, Mucci F, Dell'Osso L. 2020. Vitamin D: A Pleiotropic Hormone with Possible Psychotropic Activities. Curr Med Chem 9. $10.2174 / 0929867328666201210104701$.

Liu MY, Yin CY, Zhu LJ, Zhu XH, Xu C, Luo CX, Chen H, Zhu DY, Zhou QG. 2018. Sucrose preference test for measurement of stress-induced anhedonia in mice. Nat Protoc 13. 10.1038/s41596-018-0011-z.

Park EH, Jung MH. 2019. The impact of major depressive disorder on adaptive function: A retrospective observational study. Medicine (Baltimore) 98. 10.1097/MD.0000000000018515.

Sa M, Ying L, Tang AG, Xiao LD, Ren YP. 2012. Simultaneous determination of tyrosine, tryptophan and 5hydroxytryptamine in serum of MDD patients by high performance liquid chromatography with fluorescence detection. Clin Chim Acta 14. 10.1016/j.cca.2012.02.019.

Song S, Pan Y, Li H, Zhen H. 2020. MiR-1202 Exerts Neuroprotective Effects on OGD/R Induced Inflammation in HM Cell by Negatively Regulating Rab1a Involved in TLR4/NF-кB Signaling Pathway. Neurochem Res 45. 10.1007/s11064-020-02991-7.

Mantle TJ, Houslay MD, Garrett NJ, Tipton KF. 1976. 5-Hydroxytryptamine is a substrate for both species of monoamine oxidase in beef heart mitochondria. $J$ Pharm Pharmacol 28. 10.1111/j.20427158.1976.tb02833.x. 
411 von der Brelie C, Waltereit R, Zhang L, Beck H, Kirschstein T. 2006. Impaired synaptic plasticity in a rat model of tuberous sclerosis. Eur J Neurosci 23. 10.1111/j.1460-9568.2006.04594.x.

Wang YC, Li X, Shen Y, Lyu J, Sheng H, Paschen W, Yang W. 2020. PERK (Protein Kinase RNA-Like ER Kinase) Protein Synthesis. Stroke 51. 10.1161/STROKEAHA.120.029071.

Yang F, Diao X, Wang F, Wang Q, Sun J, Zhou Y, Xie J. 2020. Identification of Key Regulatory Genes and Pathways in Prefrontal Cortex of Alzheimer's Disease. Interdiscip Sci 12. 10.1007/s12539-019-00353-8.

Lu Y, Ho CS, McIntyre RS, Wang W, Ho RC. 2018. Agomelatine-induced modulation of brain-derived neurotrophic factor (BDNF) in the rat hippocampus. Life Sci 1. 10.1016/j.lfs.2018.09.003.

Lu Y, Ho CS, McIntyre RS, Wang W, Ho RC. 2018. Effects of vortioxetine and fluoxetine on the level of Brain Derived Neurotrophic Factors (BDNF) in the hippocampus of chronic unpredictable mild stress-induced

Yao Q, Wu Q, Xu X, Xing Y, Liang J, Lin Q, Huang M, Chen Y, Lin B, Chen W. 2020. Resveratrol Ameliorates Systemic Sclerosis via Suppression of Fibrosis and Inflammation Through Activation of SIRT1/mTOR Signaling. Drug Des Devel Ther 2. 10.2147/DDDT.S281209.

Yi Z, Li Z, Yu S, Yuan C, Hong W, Wang Z, Cui J, Shi T, Fang Y. 2012. Blood-based gene expression profiles models for classification of subsyndromal symptomatic depression and major depressive disorder. PLoS One 7. 10.1371/journal.pone.0031283.

Zhang YQ, Wang XB, Xue RR, Gao XX, Li W. 2019. Ginsenoside Rg1 attenuates chronic unpredictable mild stressinduced depressive-like effect via regulating NF-אB/NLRP3 pathway in rats. Neuroreport 4. 10.1097/WNR.0000000000001302.

Zhang Z, Cai X, Yao Z, Wen F, Fu Z, Zhang J, Zhong Z, Huang Y, Qu S. 2020. EA Ameliorated Depressive Behaviors in CUMS Rats and Was Related to Its Suppressing Autophagy in the Hippocampus. Neural Plast. $10.1155 / 2020 / 8860968$.

Zhuo J, Chen B, Sun C, Jiang T, Chen Z, Liu Y, Nie J, Yang H, Zheng J, Lai X, Su Z, Li C, Li Y. 2020. Patchouli alcohol protects against chronic unpredictable mild stress-induced depressant-like behavior through inhibiting excessive autophagy via activation of mTOR signaling pathway. Biomed Pharmacother 127. 
Figure 1

Screening of differentially expressed genes.

Figure 1 Screening of differentially expressed genes. (A) Heatmap showing the differential expression of MRNA between four MDD patients and four healthy controls. Highly expressed mRNAs are showed in red and lowly expressed mRNAs in green. (B) Differentially expressed genes are represented as a Volcano plot $(P$-value $<0.001)$.

A

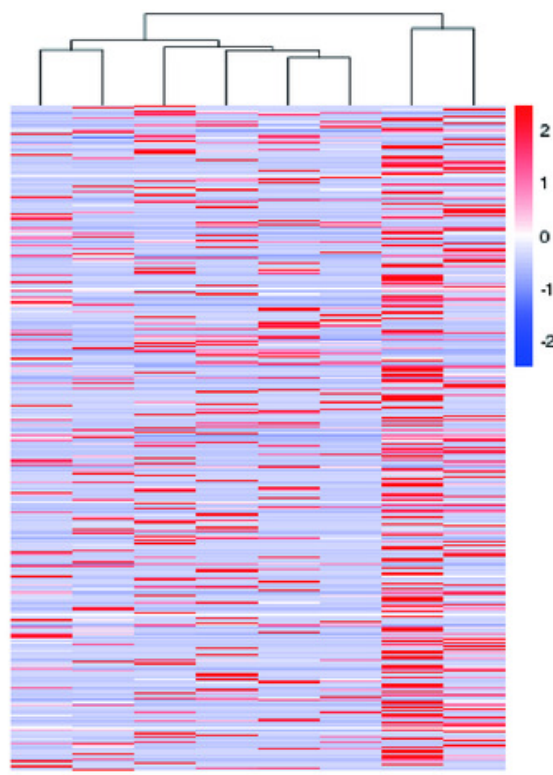

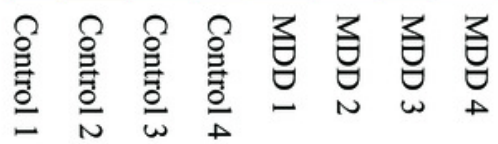

B

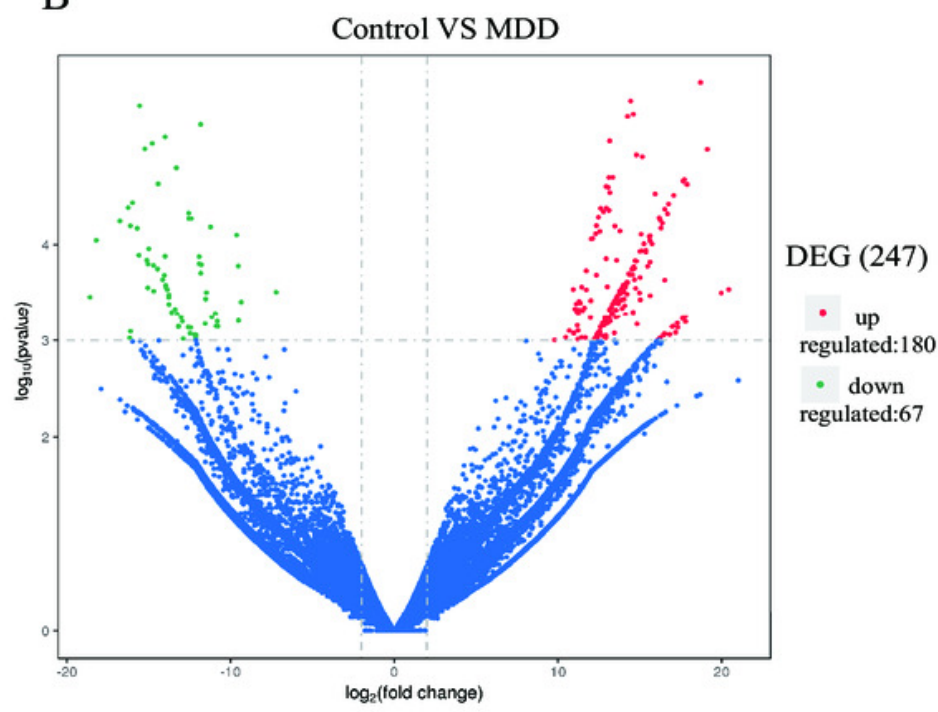




\section{Figure 2}

GO and KEGG enrichment analyses of differentially expressed genes (DEGs) between the control blood samples and the MDD blood samples.

Figure 2 GO and KEGG enrichment analyses of differentially expressed genes (DEGs) between the control blood samples and the MDD blood samples. (A) GO Functional Enrichment Map of DEGs; blue represented biological process; brown represented cellular component; green represented molecular function (Count; $p$-value $<0.05$ ). (B) The first nine significantly enriched pathways of 247 DEGs were shown by their scores (Count ; $p$ value $<0.05)$. 
A

negative regulation of transcription from RNA polymerase II promoter

BP

positive regulation of GTPase activity

intracellular signal transduction

cell-cell adhesion

vesicle-mediated transpor

regulation of small GTPase mediated signal transduction

peptidyl-serine phosphorylation

positive regulation of myelination

cytoplasm

cytosol

nucleoplasm

membrane

cell-cell adherens junction

cytoskeletor

protein binding

cadherin binding involved in cell-cell adhesion

protein ser ine/threonine kinase activit:

protein kinase activity

enzyme binding

GTPase activator activity

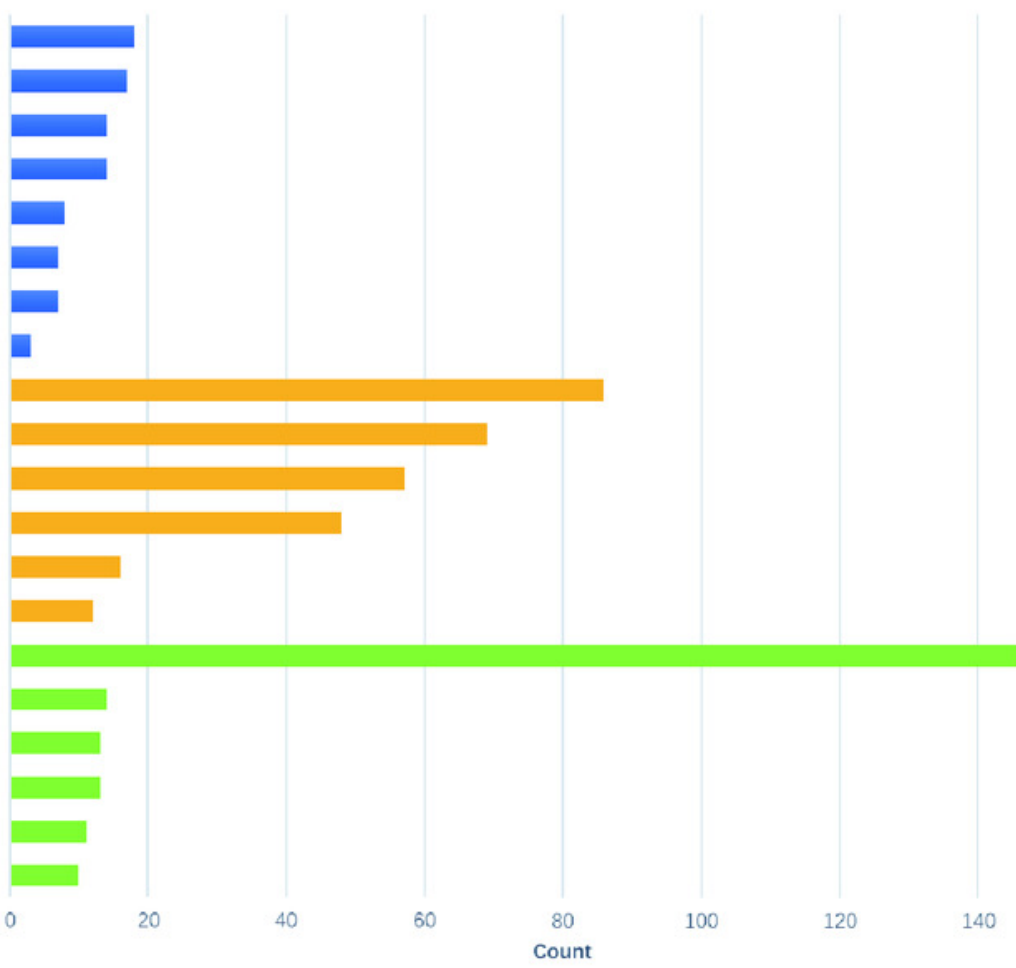

B

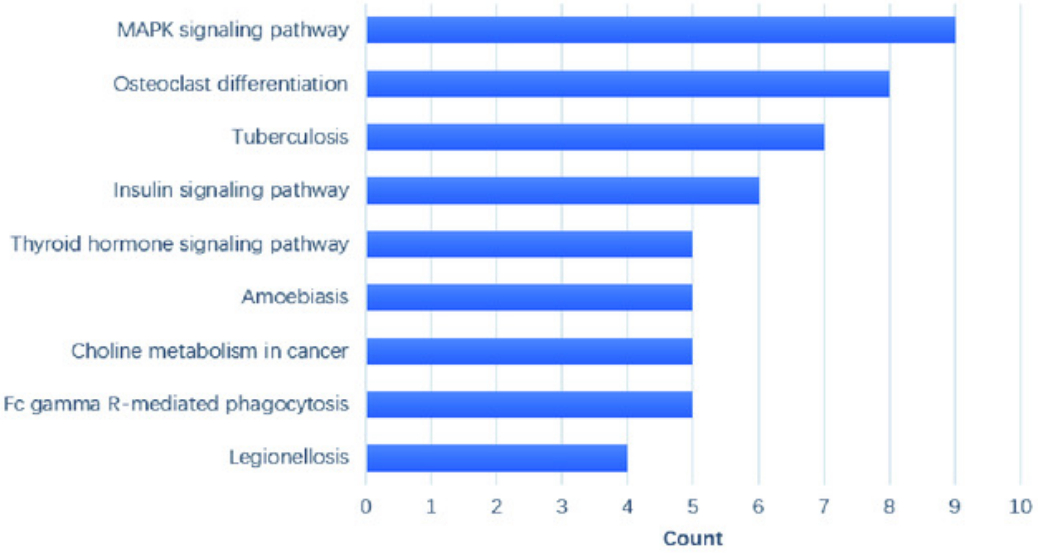




\section{Figure 3}

Protein-protein interaction (PPI) network construction and analysis of the most significant module of differentially expressed genes (DEGs).

Figure 3 Protein-protein interaction (PPI) network construction and analysis of the most significant module of differentially expressed genes (DEGs). (A) The PPI network of DEGs was constructed in STRING database (https://string-db.org/) and visualized in Cytoscape. Highly expressed mRNAs are showed in red and lowly expressed mRNAs in green, the unchanged mRNAs in blue. (B) The most significant module was obtained from the PPI network by using cytoHubba plugin. (C) The first 10 significantly enriched pathways of 10 hub genes are shown by their scores (Count; $P$-value $<0.05$ ). 


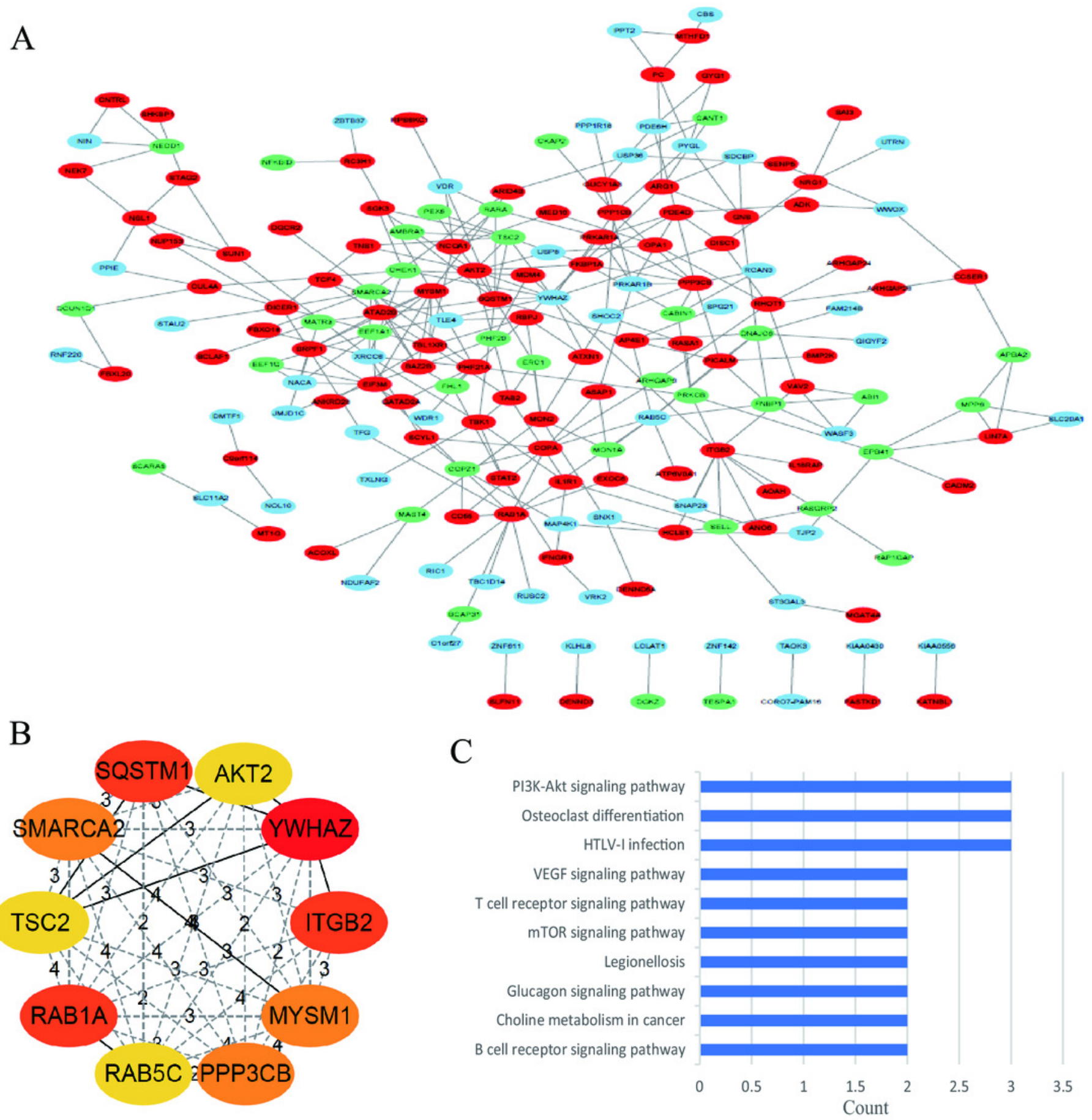


Figure 4

Quantitative real-time PCR analysis of the differences in expression of mRNA of 10 hub genes between controls and patients with major depressive disorder.

Figure 4 Quantitative real-time PCR analysis of the differences in expression of mRNA of 10 hub genes between controls and patients with major depressive disorder. A total of one downregulated gene (ITGB2) and six upregulated genes (YWHAZ, SQSTM1, RAB1A, MYSM1, AKT2, TSC2) were detected; however, SMARCA2, PPP3CB and $R A B 5 C$ were not detected $* * P<0.01$.

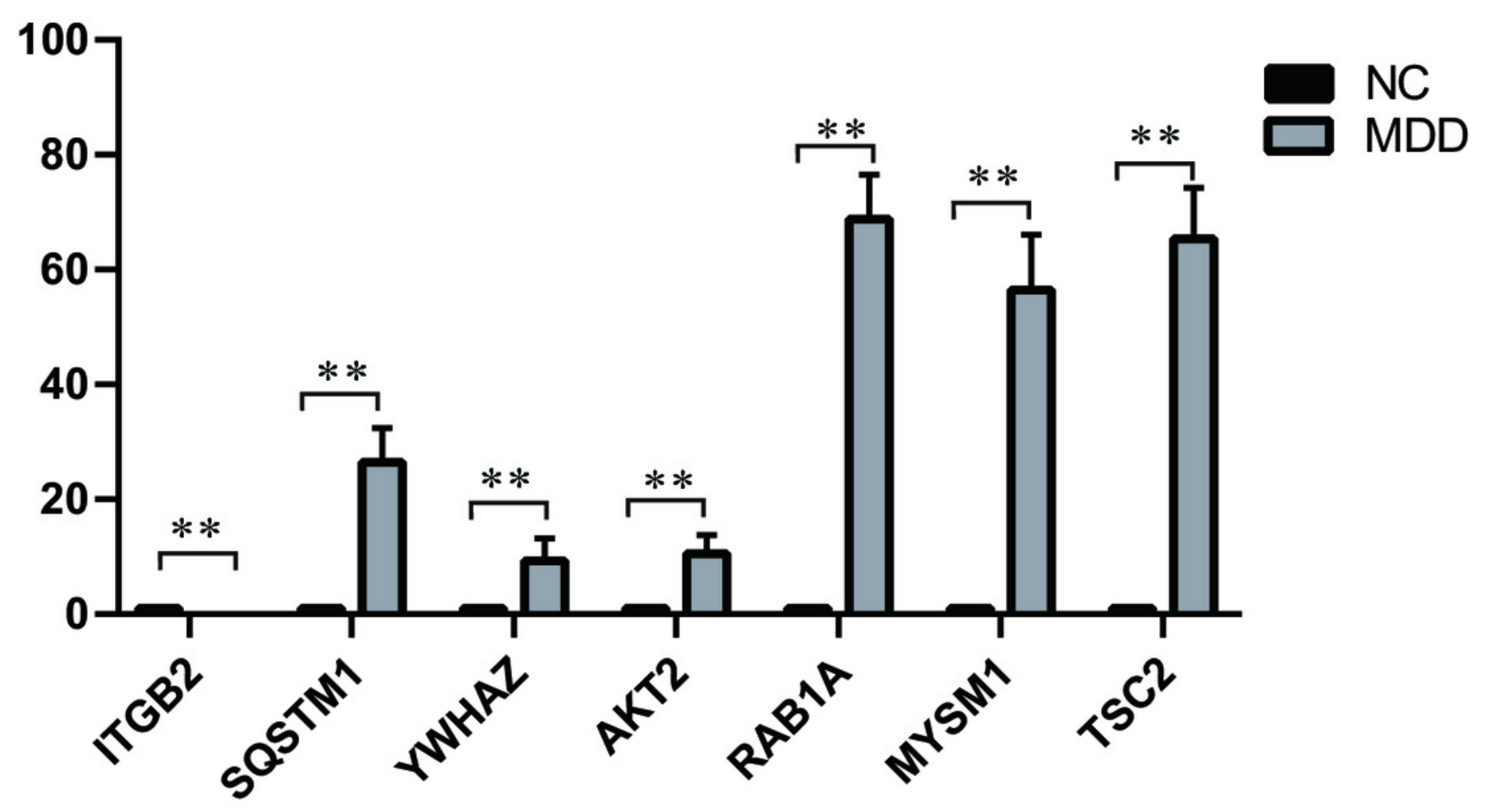


Figure 5

Quantification of SQSTM1 and TNFa levels in plasma.

Figure 5 Quantification of SQSTM1 and TNF $\alpha$ levels in plasma. (A) The protein level of SQSTM1 was increased in the plasma $(\mathrm{ng} / \mathrm{mL})$. (B) The protein level of TNF $\alpha$ was upregulated in the plasma (pg/mL). (C) A strong positive correlation was showed between SQSTM1 and TNF $\alpha$ protein $(r=0.659, P=0.00000115)$. Data are presented as mean \pm standard deviation; $\mathrm{n}=44 /$ group. ${ }^{*} * \mathrm{P}<0.01$.
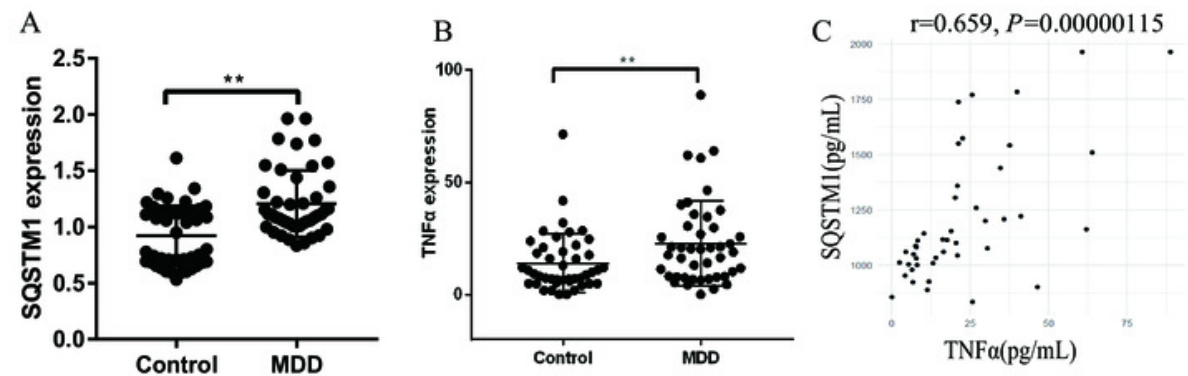


\section{Figure 6}

Autophagy related proteins were detected in CUMS rat models

Figure 6 Validation of autophagy-related protein expression in CUMS rat model.

Immunofluorescence double-labeling showed the upregulation of SQSTM1 (A) and LC3 (B) in CUMS rat models. (C, D) LC3 II and SQSTM1 protein levels were increased in CUMS rat models according to the western blot assay. (E) p-mTOR protein level was decreased in CUMS rat model as per the western blot assay. (F) TNF $\alpha$ protein levels were increased in CUMS rat models according to the western blot assay. (G)The sucrose preference values were decreased in CUMS rat models. Scale bar $=50 \mu \mathrm{m}$. Data are presented as mean \pm standard deviation; $\mathrm{n}=6$ /group. $* P<0.05, * * P<0.01$. 
A
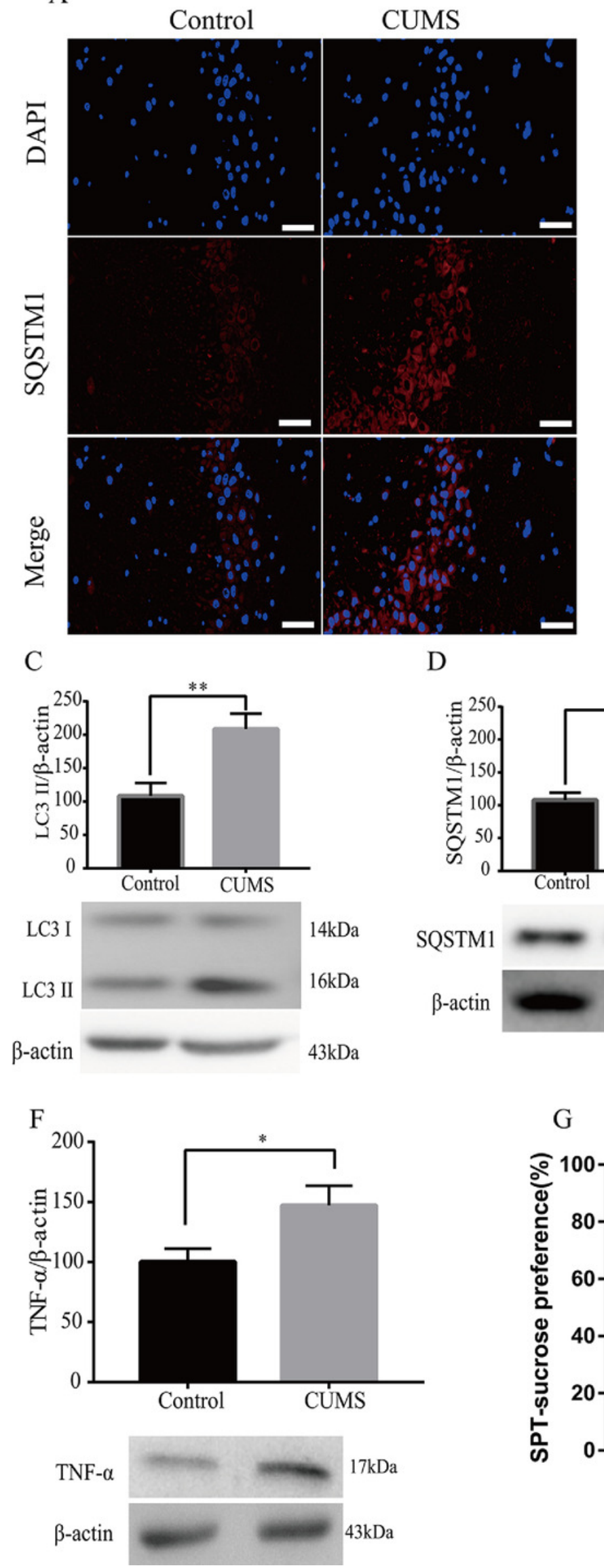

G
B

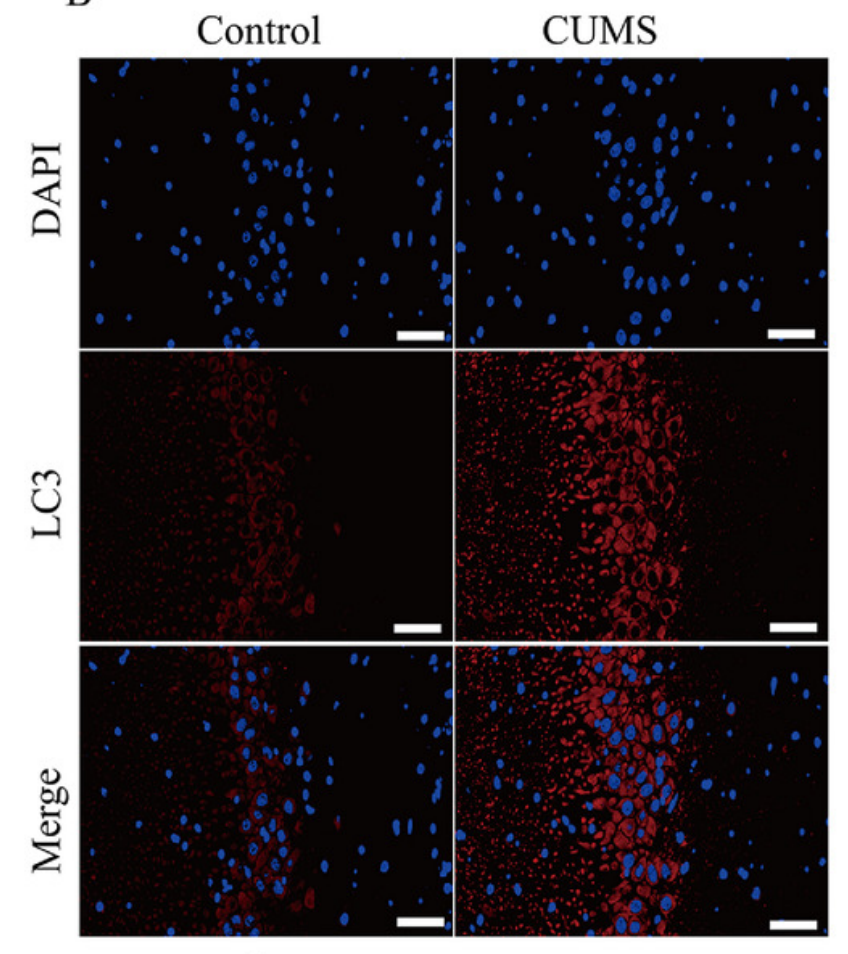

E
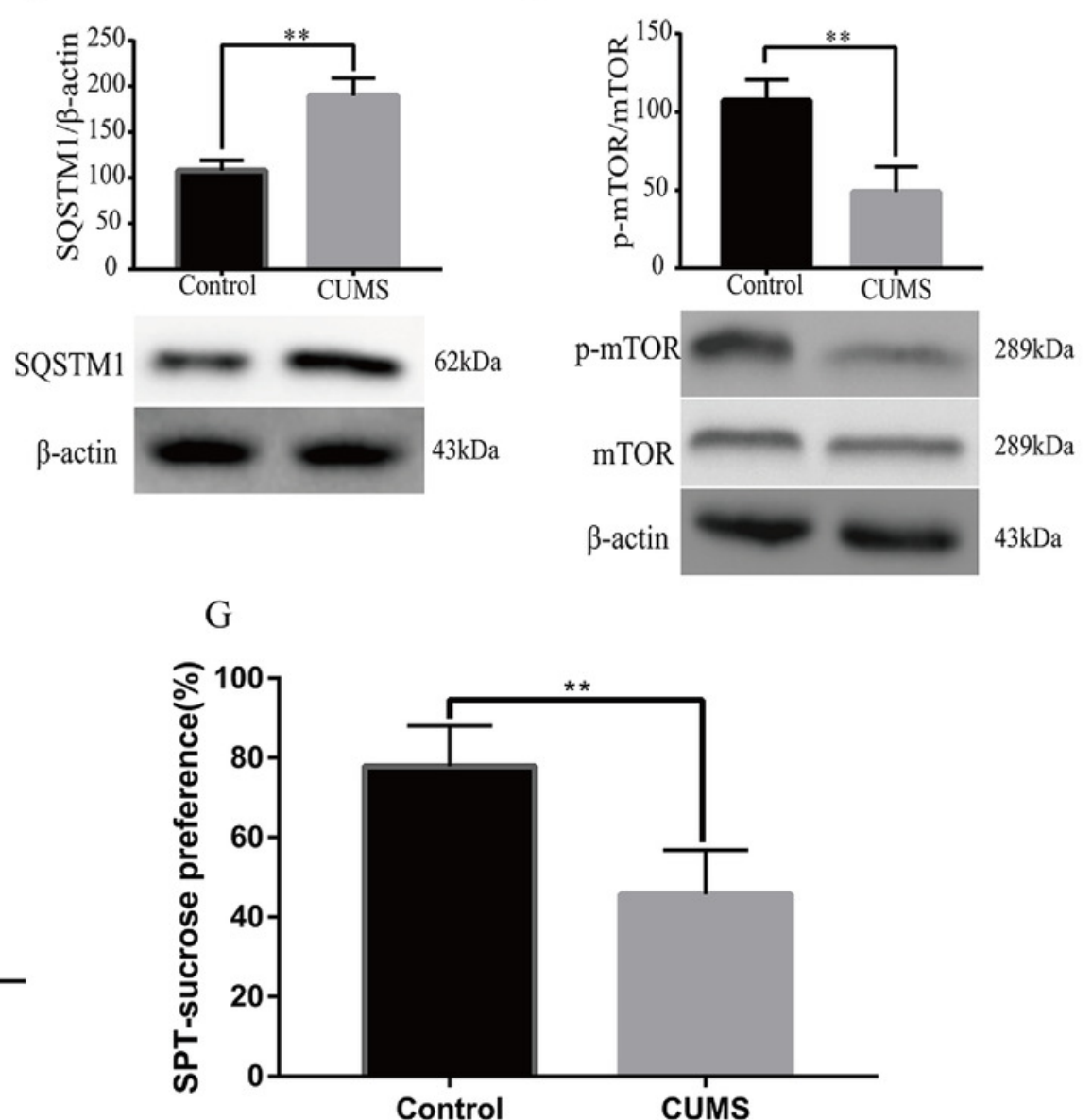

Peer) reviewing PDF | (2020:12:56643:2:2:NEW 2 Jun 2021) 
Table $\mathbf{1}$ (on next page)

Table 1. Primer sequences.

Primer sequences 
1 Table 1. Primer sequences.

\begin{tabular}{lll}
\hline Gene & Forward Primer (5'-3') & Reverse Primer (5'-3') \\
\hline YWHAZ & CTTGGAGGGTCGTCTCAAGT & GCTCTCTGCTTGTGAAGCAT \\
SQSTM1 & AATGGGTCCACCAGGAAACT & TTTCCCTCCGTGCTCCACAT \\
ITGB2 & ATCATGGACCCCACAAGCCT & GTCCATCAGATAGTACAGGT \\
RAB1A & CCCGGAACAGCCTATCTCAT & CTGAGTCGCCAATCAGAAGT \\
MYSM1 & AGCTAATTGGAAGCCGCACT & CATGATGGTGTCCATGCCT \\
SMARCA2 & ACTCTGAGAGAAGCTCGCAT & TTTGGAGAGCTTCTGGATCT \\
PPP3CB & AAGTAGGAGGATCACCTGCT & AAGGTGTCTGCATTCATGGT \\
AKT2 & TGTCATACGCTGCCTGCAGT & GAGCCACACTTGTAGTCCAT \\
TSC2 & ATCAAGGTGCTGGACGTGCT & TGCCAGGTCCACCAGCAACT \\
RAB5C & CTGCTGGGAACAAGATCTGT & CCCAGATCTCAAACTTGACT \\
HPRT & ATGGCGACCCGCAGCCCT & CCATGAGGAATAAACACCCT \\
\hline
\end{tabular}


Table 2 (on next page)

Table 2 Functional roles of 10 hub genes.

Table 2 Functional roles of 10 hub genes. 
Table 2 Functional roles of 10 hub genes.

\begin{tabular}{|c|c|c|c|}
\hline NO. & Gene Name & Description & Function \\
\hline 1 & YWHAZ & $\begin{array}{l}\text { tyrosine 3- } \\
\text { monooxygenase/tryptophan } \\
\text { 5-monooxygenase activation } \\
\text { protein zeta }\end{array}$ & $\begin{array}{l}\text { regulates spine maturation through the modulation of } \\
\text { ARHGEF7 activity }\end{array}$ \\
\hline 2 & SQSTM1 & sequestosome 1 & $\begin{array}{l}\text { Autophagy receptor required for selective } \\
\text { macroautophagy (aggrephagy), May regulate the } \\
\text { activation of NFKB1 by TNF-alpha, nerve growth } \\
\text { factor (NGF) and interleukin-1. }\end{array}$ \\
\hline 3 & ITGB2 & integrin subunit beta 2 & $\begin{array}{l}\text { Integrin ITGAL/ITGB2 in association with ICAM3, } \\
\text { contributes to apoptotic neutrophil phagocytosis by } \\
\text { macrophages }\end{array}$ \\
\hline 4 & RAB1A & $\begin{array}{l}\text { RAB1A, member RAS } \\
\text { oncogene family }\end{array}$ & $\begin{array}{l}\text { RAB1A regulates vesicular protein transport from the } \\
\text { endoplasmic reticulum (ER) to the Golgi compartment } \\
\text { and on to the cell surface,and plays a role in IL- } 8 \text { and } \\
\text { growth hormone secretion }\end{array}$ \\
\hline 5 & MYSM1 & $\begin{array}{l}\text { Myb like, SWIRM and } \\
\text { MPN domains } 1\end{array}$ & $\begin{array}{l}\text { Acts as a coactivator by participating in the initiation } \\
\text { and elongation steps of androgen receptor (AR)- } \\
\text { induced gene activation }\end{array}$ \\
\hline 6 & SMARCA2 & $\begin{array}{l}\text { SWI/SNF related, matrix } \\
\text { associated, actin dependent } \\
\text { regulator of chromatin, } \\
\text { subfamily a, member } 2\end{array}$ & $\begin{array}{l}\text { Involved in transcriptional activation and repression of } \\
\text { select genes by chromatin remodeling (alteration of } \\
\text { DNA-nucleosome topology) }\end{array}$ \\
\hline 7 & РPР3CB & $\begin{array}{l}\text { protein phosphatase } 3 \\
\text { catalytic subunit beta }\end{array}$ & $\begin{array}{l}\text { Dephosphorylates and inactivates transcription factor } \\
\text { ELK1 }\end{array}$ \\
\hline 8 & AKT2 & $\begin{array}{l}\text { AKT serine/threonine kinase } \\
2\end{array}$ & $\begin{array}{l}\text { AKT2 regulates many processes including metabolism, } \\
\text { proliferation, cell survival, growth and angiogenesis. }\end{array}$ \\
\hline 9 & $\mathrm{TSC} 2$ & TSC complex subunit 2 & $\begin{array}{l}\text { a direct activator of the protein kinase activity of } \\
\mathrm{mTORC} 1\end{array}$ \\
\hline 10 & RAB5C & $\begin{array}{l}\text { RAB5C, member RAS } \\
\text { oncogene family }\end{array}$ & $\begin{array}{l}\text { RAB5C, Ras oncogene superfamily member,involved } \\
\text { in endocytosis }\end{array}$ \\
\hline
\end{tabular}

